\title{
An investigation of honey bee virus prevalence in managed honey bees (Apis mellifera and Apis cerana) undergone colony losses: a case study in China
}

\author{
Yanchun Deng \\ Institue of Apicultural Research, CAAS \\ Li Zhang \\ Institute of Apicultural Research, CAAS \\ Xuejian Jiang \\ Institute of forestry protection \\ Sa Yang \\ Institute of Apicultural Research, CAAS \\ Shuai Deng \\ Institute of Apicultural Research, CAAS \\ Chunsheng Hou ( $\nabla$ houchunsheng@caas.cn )
}

Research note

Keywords: Honey bee viruses, BQCV, CBPV, AmFV, CSBV

Posted Date: August 3rd, 2020

DOI: https://doi.org/10.21203/rs.3.rs-48932/v1

License: (c) (i) This work is licensed under a Creative Commons Attribution 4.0 International License. Read Full License 


\section{Abstract}

Objective: In the absence of known clinical symptoms, viruses were considered to be the most probable key pathogens of honey bee. Therefore, the aim of this study was to investigate the prevalence and distribution of honey bee viruses in managed Apis mellifera and Apis cerana in China.

Results: We conducted a screening of 9 honey bee viruses on $A$. mellifera and $A$. cerana samples collected from 54 apiaries from 13 provinces in China. We found that the types and numbers of viruses significantly differed between A. mellifera and A. cerana. Black queen cell virus (BQCV), chronic bee paralysis virus (CBPV), Apis mellifera filamentous virus (AmFV) and Kakugo virus (DWV-C/KV) were the primary viruses found in $A$. mellifera colonies, whereas Chinese sacbrood bee virus (CSBV) and sacbrood bee virus (SBV) were the primary viruses found in A. cerana. The percentage infection of BQCV and CSBV were $84.6 \%$ and $61.6 \%$ in all detected samples. We first detected the occurrences of Varroa destructor virus-1(VDV-1, DWV-B) and DWV-C/KV in China but not ABPV in both $A$. mellifera and $A$. cerana.

\section{Introduction}

Viruses, bacteria, fungi and parasites were all confirmed to be capable of inducing reductions in colony populations [1-4]. Among these pathogens, more than 20 viruses were identified to infect honey bee, most of them belonged to the family Picornaviridae and possessed a single-strand positive-sense RNA genome. These viruses were demonstrated to not only have severe impacts on individual bees by damaging their physiology [2], interfering with their productivity [5] and contributing to behavioral disorders [6] but also on the colony growth rate and colony fitness (i.e., decrease of foraging activity at the colony level [7]).

However, most honey bee viruses cause covert infections without significant clinical symptoms, which is a barrier to their study. A few viruses can be recognized during the late stage of infection by their appearance, such as chronic bee paralysis virus (CBPV), black queen cell virus (BQCV), deformed wing virus (DWV), sacbrood virus (SBV) and Israeli acute paralysis virus (IAPV). Nevertheless, little is known about DNA viruses of honey bees compared to RNA viruses. Recently, the DNA virus Apis mellifera filamentous virus (AmFV) was reported to be associated with crawling bees. The AmFV viral loads were reported to be higher in the bees in spring in Switzerland and demonstrated a significant correlation with RNA viruses in infected honey bees [8]. Owing to the constant discovery of emerging viruses, many investigations have reported the occurrence and prevalence of honey bee viruses as well as other viruses that may infect honey bees, especially since IAPV was found to be associated with colony collapse disorder (CCD) [9]. Ai et al. [10] investigated the occurrence of 7 honey bee viruses in 2011 in China, and found that DWV was the most prevalent of the viruses. Subsequently, a report that demonstrated the prevalence of 6 viruses in A. cerana found that BQCV had an infection rate of $98 \%$ in $A$. cerana [11]. In contrast, Yang et al. [12] found that DWV was the most prevalent among the 7 viruses in China regardless of whether $A$. mellifera or $A$. cerana was investigated. 
Despite the prevalence of honey bee viruses that have been extensively investigated around the world, several neglected and new honey bee viruses have not been described in China. To obtain a broader spectrum of information about honey bee viruses, we investigated the presence of viruses in A. mellifera and $A$. cerana bee samples from different provinces that reported large bees crawling in front of the hives and undergone colony losses. We screened bee samples from 56 apiaries from 13 provinces to identify the presence and distribution of 9 honey bee viruses in China. We found that BQCV and CSBV were the most prevalent in $A$. mellifera and $A$. cerana, respectively. Additionally, we discovered that a high percentage of colonies were infected by AmFV and DWV-C/KV in many regions in China for the first time.

\section{Methods}

\section{Bee samples}

Honey bees were sampled from 56 apiaries in 13 provinces from March to November, 2015. Worker samples of $A$. mellifera were collected from 38 apiaries in 8 provinces and samples of $A$. cerana were obtained from 18 apiaries in 10 provinces (Table S1). Ten colonies were randomly selected in each apiary per province. Clinical signs of virus infection were not found in these colonies; thus, these colonies were thought to be capable of honey production, although the colonies were reported to have undergone large losses or were identified with crawling bees. All of the above colonies were maintained consistent with guidelines for beekeeping practice manipulation and regularly monitored and treated for mites (only for $A$. mellifera).

\section{DNA and RNA extraction}

Briefly, for the detection of Apis mellifera filamentous virus (AmFV), total DNA was isolated from pooled honey bee samples ( 50 bees) with the TissuePrep homogenizer (Gening Scientific, Beijing, China). Then, genomic DNA was extracted from the filtrate using the DNA Purification Kit (Promega Corp., Madison, WI, USA) following the manufacturer's instructions. The resulting DNA was eluted into $20 \mu \mathrm{L}$ of nuclease-free water and stored at $-20^{\circ} \mathrm{C}$.

For the other 8 RNA viruses (IAPV, SBV, ABPV, BQCV, CBPV, CSBV, KBV DWV-A, DWV-B, and DWV-C/KV), total RNA was extracted from approximately 50 pooled honey bee samples using the TRIzol Kit (Invitrogen, USA) according to the manufacturer's protocol in the TissuePrep homogenizer (Gening Scientific, Beijing, China). The obtained RNA was dissolved in $20 \mu \mathrm{L}$ of sterile water and stored at $-80^{\circ} \mathrm{C}$ prior to analysis. The quantity and purity of the RNA were measured using a Nanodrop spectrophotometer (Thermo Scientific, Beijing, China). The cDNA was synthesized using the M-MLV reverse transcriptase with the oligo $\mathrm{dT}$ primer according to the manufacturer's instructions. The CDNA samples were used as templates for PCR amplification with specific primers corresponding to viral genes.

\section{PCR detection}

The primer sequences, orientation and references are provided in Table S2. For the 8 RNA viruses, the initial cycle for reverse transcription was $80^{\circ} \mathrm{C}$ for $30 \mathrm{~min}$, followed by the PCR cycles. The PCR reaction 
for AmFV and the other 10 RNA viruses consisted of a total $20 \mu$ volume containing $2 \times$ GoTaq reaction buffer (Promega, USA), $1 \mu \mathrm{M}$ of the sense and antisense primers, $1 \mu \mathrm{L}$ of CDNA or DNA, and nuclease-free water. The cycling conditions were as follows: $1 \mathrm{~min}$ at $95^{\circ} \mathrm{C} ; 33$ cycles of $30 \mathrm{~s}$ at $94^{\circ} \mathrm{C}, 30 \mathrm{~s}$ at $55^{\circ} \mathrm{C}$ and $72{ }^{\circ} \mathrm{C}$ for $1 \mathrm{~min}$; a final extension of $10 \mathrm{~min}$ at $72{ }^{\circ} \mathrm{C}$; and cooling to $4{ }^{\circ} \mathrm{C}$. The PCR amplification products were separated in a $2 \%$ agarose gel stained with GV II (Biomec, China) and photographed with a FR-200A luminescent and fluorescent biological image analysis system (Furi, China). The product size was determined using a 100-bp molecular size ladder.

\section{Statistical analysis}

The results were expressed as the presence or absence of virus in an apiary. We considered virus to be present in an apiary if samples from any colony were positive for any virus. Differences in the viral infection percentages of different provinces and virus co-infection were estimated using the Chi-square test with the SPSS 21 software.

\section{Results}

\section{Virus prevalence and occurrence}

As shown in Table 1, different types of viruses were detected in all provinces with the exception of Jiangsu province where no virus was detected. All of the viruses were present in the different provinces with the exception of KBV and ABPV, which were not detected. BQCV was the most prevalent virus and was present in $18 \%$ of the A. mellifera apiaries and in seven provinces. CSBV was found in $38.9 \%$ of the A. cerana apiaries and in seven provinces. In contrast, BQCV was detected in $22 \%$ of the $A$. cerana apiaries and CSBV was found in $2.6 \%$ of the $A$. mellifera apiaries. The following frequencies were obtained for the remainder of the viruses: IAPV was detected in $7.9 \%$ of the A. mellifera apiaries and 5.6 $\%$ of the $A$. cerana apiaries; CBPV was found in $15.8 \%$ of the $A$. mellifera apiaries and was not detected in A. cerana; SBV was identified in $5.3 \%$ of the $A$. mellifera apiaries and $16.7 \%$ of the $A$. cerana apiaries; DWV-A was found in $13.2 \%$ of the $A$. mellifera apiaries and $5.6 \%$ of the A. cerana apiaries; DWV-B was found in $10.5 \%$ of the $A$. mellifera apiaries and was not detected in the A. cerana apiaries; AmFV was found in $13.2 \%$ of the $A$. mellifera apiaries and $11.1 \%$ of the $A$. cerana apiaries; and DWV-C/KV was found in $10.5 \%$ of the $A$. mellifera apiaries and $11.1 \%$ of the $A$. cerana apiaries.

BQCV was the most common virus and accounted for $84.6 \%$ among the tested samples in each province (Figure 1A). The second highest frequency virus was CSBV (61.6\%), followed by another 3 viruses at equal frequency (53.8\%; DWV-A, AmFV and DWV-C/KV). The least frequent viruses were IAPV and DWV-B with $30.8 \%$. The number of honey bee viruses in A. mellifera was higher than that in A.cerana in the same province (Figure 1B) with the exception of Chongqing and Yunnan, which gave priority to keeping $A$. cerana. The number of viruses in $A$. mellifera in Zhejiang was the same as that of $A$. cerana. Liaoning province had the highest number of viruses, with 9 viruses in $A$. mellifera and only one in $A$. cerana. Similarly, there were 7 viruses in $A$. mellifera in Inner Mongolia and Heilongjiang and no viruses were detected in A. cerana. 


\section{Co-infection of viruses in each province}

The co-occurrence of more than one virus in one province was fairly common in both the A. mellifera and A. cerana apiaries (Table S3 and Table S4). In A. mellifera, virus co-infection varied from 2 to 6 viruses. Co-infection with 2 viruses represented $28.9 \%$ of the occurrences, 3 viruses represented $31.6 \%$, 4 viruses represented $15.7 \%, 5$ viruses represented $10.5 \%$ and 6 viruses represented $7.89 \%$. Among the multiple co-infections, BQCV and CBPV co-infection was the most common 2 virus co-infection and accounted for $10.53 \%$. IAPV, SBV and CBPV was the highest percentage 3 virus co-infection with $7.89 \%$, followed by BQCV, CBPV and AmFV, IAPV, SBV and CSBV, and IAPV, DWV-A and DWV-B. IAPV, BQCV, DWV-A and DWV$B$ was the most common 4 virus co-infection with $13.16 \%$. In contrast, fewer co-infections were detected in A. cerana compared to A. mellifera (Table S4). Virus co-infections in A. cerana primarily involved 2 and 3 viruses. Simultaneous infections with 2 viruses were detected in $61.11 \%$ and with 3 viruses in $16.67 \%$ of the apiaries. The most prevalent co-infection was SBV and CSBV in A. cerana, accounting for $22.22 \%$. Co-infection with 4 viruses (IAPV, BQCV, DWV-A, and DWV-B) was significantly higher than the other co-

infections in A. mellifera, $\left(\mathrm{X}^{2}=12.61, \mathrm{P}=0.00038, \mathrm{df}=1\right)$ (Table S5), whereas in A. cerana co-infection with 2 viruses was significantly more common than the other co-infection types (e.g., BQCV and CSBV; $\left.\chi^{2}=0.0009, P=0.02, d f=1\right)($ Table S6).

\section{Discussion}

The results showed that several viruses with a high prevalence and wide distribution in China were not previously reported, such as DWV-B, DWV-C/KV and AmFV. Our surveys indicated that DWV-C/KV and AmFV were widely distributed in China in both A. mellifera and A. cerana. Additionally, neither ABPV nor $\mathrm{KBV}$ was found in any province. This primary investigation helps to narrow future efforts to identify the cause of colony decline.

In addition, we found multiple virus co-infections in honey bee colonies of both $A$. mellifera and $A$. cerana. The widespread distribution of honey bee viruses in the two honey bee species suggests a stable interaction between the viruses and their hosts. Simultaneous infection with more than 3 viruses (65.78\%) occurred in more than 12 apiaries of $A$. mellifera, which was more than occurred in A. cerana, suggesting that multiple infections were very common in A. mellifera; in contrast, most cases of virus coinfection in $A$. cerana involved 2 viruses. Data from experiment studies indicated that multiple virus infections will increase the virulence [13]. A recent report showed that IAPV could be elevated to a higher level when several viruses were mixed to infect honey bees, although the initial IAPV infection level was not the highest [14]. Thus, colony losses might be due to a combination of multiple viruses.

\section{Conclusion}

We demonstrated for the first time the presence of DWV-B and DWV-C/KV. The most prevalent viruses were $\mathrm{BQCV}, \mathrm{CBPV}, \mathrm{DWV}-\mathrm{C} / \mathrm{KV}$ and $\mathrm{AmFV}$ in A. mellifera and CSBV and SBV in A. cerana. The results of 
this study suggest that future studies should focus on these viruses to narrow our objectives and help us understand the differences in different areas and hosts.

\section{Limitations}

A main limitation of current study is that we did not investigate the honey bee samples from all provinces. Therefore, we cannot draw conclusions regarding the prevalence of honey bee viruses in China. In addition, the differences in the distributions and levels of different viruses among the different tissues of honey bees also require further study, although multiple virus co-infections were found.

\section{Declarations}

\section{Availability of data and materials}

All data generated or analysed during this study are included in this published article and Additional file.

Ethics approval and consent to participate

Not applicable.

Consent for publication

Not applicable.

Competing interests

Authors have no potential conflict or competing interests to declare.

\section{Funding}

We acknowledge the support of the Fundamental Research Funds for CAAS (Y2020PT17), the Agricultural Science, Technology Innovation Program of CAAS (CAAS-ASTIP-2020-IAR), The Key Technology Research and Development Program of Guangxi province (AB16380094).

\section{Authors' contributions}

CSH conceived and designed the experiments. YCD, LZ, XJJ, SY and SD performed the experiments. CSH analyzed the data and wrote the manuscript.

\section{Acknowledgements}


We acknowledge the assistance of beekeepers from selected apiaries from 13 provinces.

\section{References}

1. Garcia-Gonzalez E, Genersch E. Honey bee larval peritrophic matrix degradation during infection with Paenibacillus larvae, the aetiological agent of American foulbrood of honey bees, is a key step in pathogenesis. Environ Microbiol. 2013;15:2894-901. doi:10.1111/1462-2920.12167.

2. Locke B, Forsgren E, Fries I, de Miranda JR. Acaricide treatment affects viral dynamics in Varroa destructor-Infested honey bee colonies via both host physiology and mite control. Appl Environ Microb. 2012;78:227-35. doi:10.1128/aem.06094-11.

3. Hou C, Rivikin H, Slabezki Y, Chejanovsky N. Dynamics of the Presence of Israeli Acute Paralysis Virus in Honey Bee Colonies with Colony Collapse Disorder. Viruses. 2014;6:2012-27.

4. Yang $X$, Cox-Foster D. Effects of parasitization by Varroa destructor on survivorship and physiological traits of Apis mellifera in correlation with viral incidence and microbial challenge. Parasitology. 2007;134:7.

5. Ivan M, Dirk de G, Felix W, Guy S. Effect of oral infection with Kashmir bee virus and Israeli acute paralysis virus on bumblebee (Bombusterrestris) reproductive success. J.Inverteb. Pathol. 5, (2014).

6. Li Z, et al. Viral infection affects sucrose responsiveness and homing ability of forager Honey Bees Apis mellifera L. PLoS ONE. 2013;8:e77354. doi:10.1371/journal.pone.0077354.

7. Simon-Delso N, et al. Honeybee colony disorder in crop areas: The Role of Pesticides and Viruses. PLoS ONE. 2014;9:e103073. doi:10.1371/journal.pone.0103073.

8. Hartmann UF, et al. Dynamics of Apis mellifera filamentous virus (AmFV) infections in honey bees and relationships with other parasites. Viruses. 2015;7:13.

9. Forgách P, Bakonyi T, Tapaszti Z, Nowotny N, Rusvai M. Prevalence of pathogenic bee viruses in Hungarian apiaries: Situation before joining the European Union. JInvertebr Pathol. 2008;98:235-8. doi:http://dx.doi.org/10.1016/j.jip.2007.11.002.

10. Li J, et al. The prevalence of parasites and pathogens in Asian honeybees Apis cerana in China. PLoS ONE. 2012;7:e47955. doi:10.1371/journal.pone.0047955.

11. Yang B, Peng G, Li T, Kadowaki T. Molecular and phylogenetic characterization of honey bee viruses, Nosema microsporidia, protozoan parasites, and parasitic mites in China. Ecol Evol. 2013;3:298311. doi:10.1002/ece3.464.

12. Fujiyuki T, et al. Novel insect picorna-like virus identified in the brains of aggressive worker honeybees. JVirol. 2004;78:1093-100. doi:10.1128/JVI.78.3.1093-1100.2004.

13. Esmaeil A, Marina M, Steen Lykk LN, Per K. Four categories of viral infection describe the health status of honey bee colonies. PLoS ONE. 2015;10:e0140272. doi:10.1371/journal.pone.0140272.

14. $10.1038 /$ srep22265

Carrillo-Tripp J. et al. In vivo and in vitro infection dynamics of honey bee viruses. Scientific Reports 6, 22265, doi:10.1038/srep22265 (2016). 


\section{Tables}

Table 1 Presence of IAPV, BQCV, CBPV, ABPV, SBV, CSBV, DWV-A, DWV-B, AmFV, KBV and DWV-C/KV in A. mellifera and $A$. cerana samples from 56 apiaries

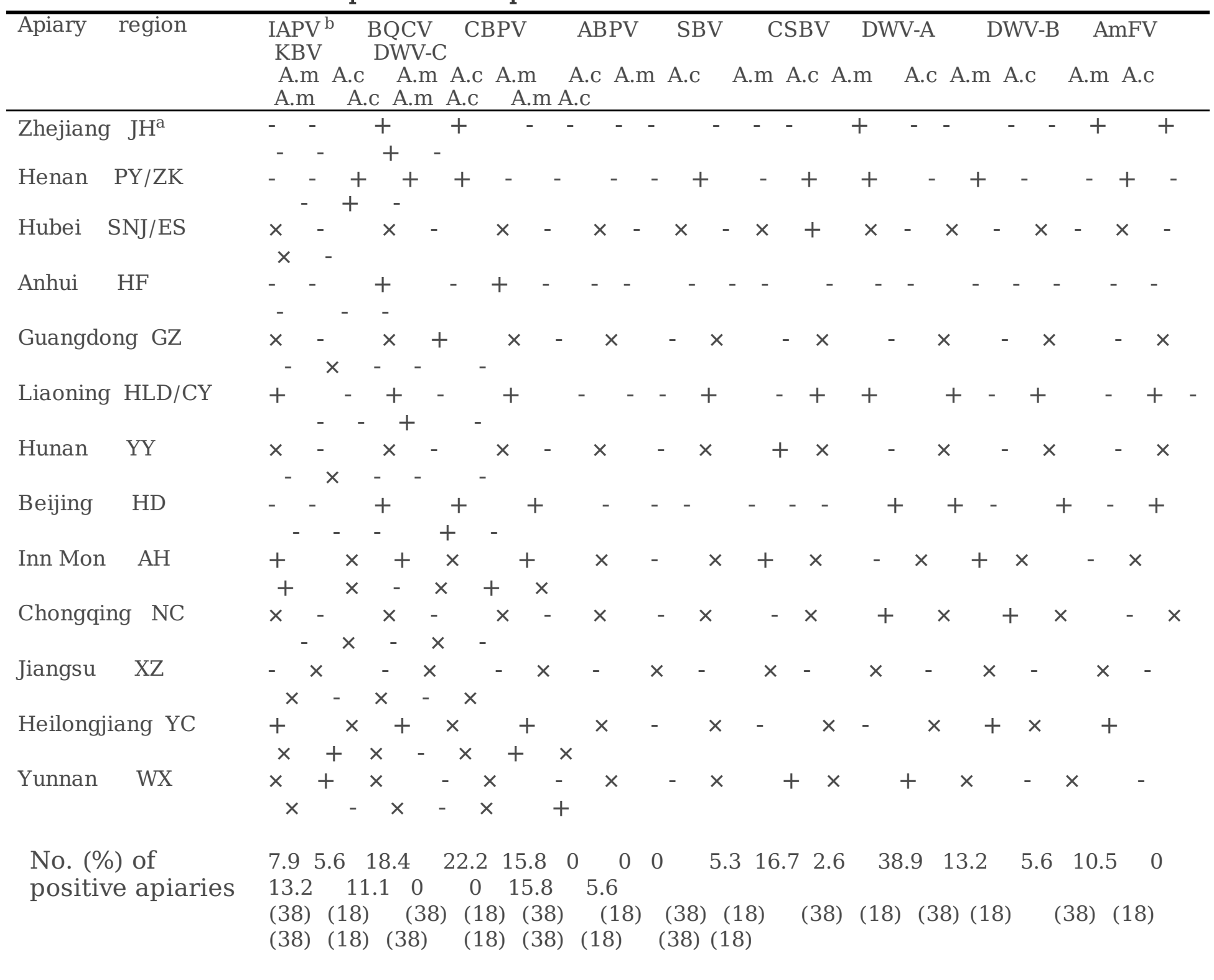

a The collection locations: JH, Jinhua; PY/ZK, Pingyu and Zhoukou; SNJ/ES, Shengnongjia and Enshi; HF, Hefei; GZ, Guangzhou; HLD/CY, Huludao and Chaoyang; YY, Yueyang; HD, Haidian; AH, Aohan; NC, Nanchuan; XZ, Xuzhou; YC, Yichun; WX, Weixi. ${ }^{b}+$, positive; -, negative; $x$, samples not available 


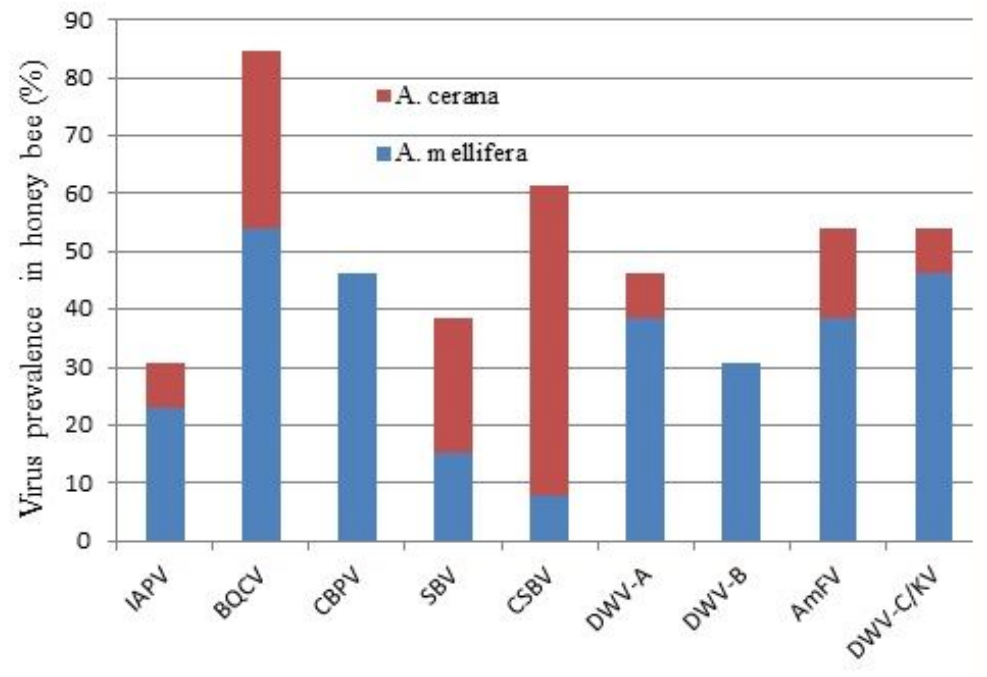

A

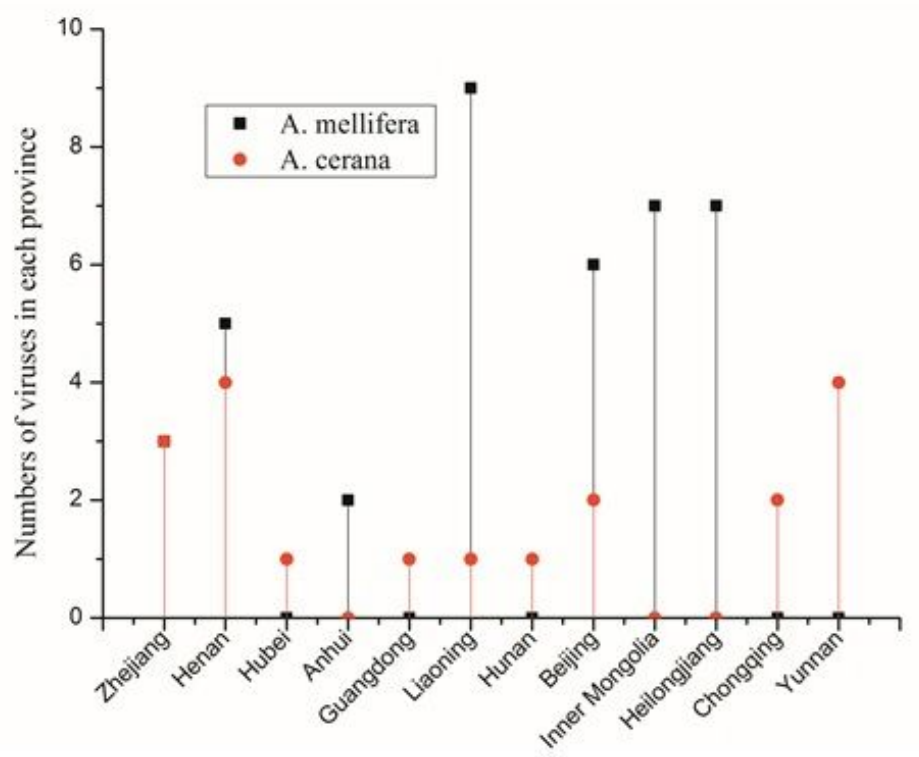

B

\section{Figure 1}

Virus prevalence varied in different honey bee species and provinces. The cumulative prevalence of different viruses in $A$. mellifera and $A$. cerana $(A)$; the number of virus in $A$. mellifera and $A$. cerana in each province (B).

\section{Supplementary Files}

This is a list of supplementary files associated with this preprint. Click to download.

- supplenmentarydataset.doc 